Tecnociencia, Vol. 23, $\mathrm{N}^{\circ} 1: 364-384$

Enero-Junio 2021

\title{
OBSERVACIONES BIOLÓGICAS Y CONDUCTUALES DE ALGUNOS SALTAMONTES (ORTHOPTERA: ACRIDOIDEA) DE PANAMÁ.
}

\begin{abstract}
${ }^{1}$ Lesbia I. De Gracia P. (D) , ${ }^{2,3,4}$ Alonso Santos Murgas.
${ }^{1}$ Universidad de Panamá, Centro Regional Universitario de Coclé, Departamento de Fisiología y Comportamiento Animal, Facultad de Ciencias Naturales, Exactas y Tecnología. ${ }^{2}$ Universidad de Panamá, Museo de Invertebrados G.B. Fairchild, Facultad de Ciencias Naturales, Exactas y Tecnología. ${ }^{3}$ Director Centro de Recursos Bióticos. ${ }^{4}$ Sociedad Mesoamericana para la Biología y la Conservación. e-mail: lesbia.degraciap@up.ac.pa; lesbiaidg20@gmail.com; santosmurgagasa@gmail.com; alonso.santos@up.ac.pa
\end{abstract}

\section{RESUMEN}

El presente trabajo recoge observaciones hechas tanto en campo como en el laboratorio de algunos saltamontes (Orthoptera: Acridoidea), en donde se describen aspectos sobre su biología y comportamiento. Rowell (2013), señala que, en Panamá, la Acridoidea está compuesta por 66 géneros y 108 especies; siendo la Acrididae la familia que tiene a su haber más del $65 \%$ del total de saltamontes; los cuales tienen una gran diversidad estructural, ecológica y de comportamiento. Observamos que Abracris flavolineata (DeGeer), 1773, Orphulella punctata (DeGeer), 1773 y Stenopola puncticeps (Stål), 1860, son especies que se encuentran distribuídos en casi todo el territorio nacional; además, los saltamontes estudiados se encontraron generalmente en plantas de la familia Poaceae, del cual se alimentaban. Se reportan nemátodos que los parasitan.

\section{PALABRAS CLAVES}

Biología, acrídidos, herbivoría, parásitos, planta hospedera.

\section{BIOLOGICAL AND BEHAVIORAL OBSERVATIONS OF SOME GRASSHOPPERS (ORTHOPTERA: ACRIDOIDEA) OF PANAMA.}




\begin{abstract}
The present work collects observations made both in the field and in the laboratory of some grasshoppers (Orthoptera: Acridoidea), where aspects of their biology and behavior are described. Rowell (2013) points out that, in Panama, the Acridoidea is composed of 66 genera and 108 species; being the Acrididae the family that has more than $65 \%$ of the total grasshoppers; which have a great structural, ecological and behavioral diversity. We observed that Abracris flavolineata (DeGeer), 1773, Orphulella punctata (DeGeer), 1773 and Stenopola puncticeps (Stål), 1860, they are species that are more frequently found in different collection points; furthermore, the grasshoppers studied were generally located in Poaceace and there are nematodes that parasitize.
\end{abstract}

\title{
KEY WORDS
}

Biology, acridids, herbivory, parasites, host plant.

\section{INTRODUCCIÓN}

El orden Orthoptera comprende los insectos terrestres comúnmente denominados saltamontes, tucuras, langostas, grillos, esperanzas y grillotopo, así como otros Neoptera relacionados (Cigliano \& Lance, 1998). Rowell (2013), señala que Caelifera está formada por ocho a nueve superfamilias (Tridactyloidea, Tetrigoidea, Eumastacoidea, Proscopioidea, Tanaoceroidea, Trigonopterigoidea, Pneumoroidea, Pyrgomorphoidea y Acridoidea), que comprenden más de 11,000 especies descritas en 2,500 géneros en todo el mundo. La Acridoidea, presenta el mayor número de géneros (1500) y de especies (8000) dentro del orden y contiene una extensa distribución geográfica (Cigliano \& Lance, 1998); Según Rowell (2013), la Acrididae está compuesta por 12 subfamilias, que incluyen 43 géneros y 69 especies y la Romaleidae, por 2 subfamilias, que incluyen 23 géneros y 39 especies; además indica que la Acrididae comprende más del $65 \%$ del total de saltamontes, los cuales tienen una gran diversidad estructural, ecológica y de comportamiento; en la Romaleidae, la Bactrophorinae, incluye especies tanto del sotobosque como del dosel del bosque.

Al realizar la preparación de los saltamontes (disectar el abdomen del insecto y extraerle sus tejidos blandos) para el montaje en los alfileres entomológicos, permite hacer observaciones post mortem, ya que se puede extraer bolsas o masas de huevos o parásitos si los hubiere. A través de las observaciones tanto in situ como en el laboratorio (cautiverio), podemos conocer hábitos alimenticios y conductuales, por ejemplo, si hay relaciones inter o intraespecíficas, de apareamiento, entre otros, que en nuestro país ha sido muy poco estudiado. 
Con el presente estudio se da a conocer aspectos biológicos y conductuales de algunos saltamontes (Acridoidea) de Panamá y con ello, contribuir con el conocimiento de este grupo de insectos.

\section{MATERIALES Y MÉTODOS}

Las observaciones se realizaron en diferentes giras de campo por provincias como: Bocas del Toro, Veraguas, Colón y la Comarca Guna Yala, entre los meses de octubre a febrero, desde 1999-2002. Se hicieron colectas diurnas de 8:00 a.m. a 2:00 p.m. (294 horas, dos personas), utilizando redes entomológicas manuales; cada especímen se colocó en un envase plástico (para su posterior preparación) o en bolsas ziploc con hojas de las plantas donde se encontraban; además se realizaron observaciones tanto in situ como en el laboratorio, para recabar información de los aspectos biológicos y conductuales.

El dosel del bosque se muestreó a través de la grúa del dosel de Fort Sherman, Proyecto de la grúa del dosel, Instituto Smithsonian de Investigaciones Tropicales (STRI), utilizando una red cuadrada que tiene un hueco en el centro de la malla al que se le ata un recipiente o bolsa ziploc; al golpear las ramas y hojas de la copa de los árboles con un pequeño tubo pvc, los insectos caen al en la red y de allí al recipiente.

\section{RESULTADOS Y DISCUSIÓN}

Comprender la biología y el comportamiento (etología) de la fauna existente es de gran interés y valor científico; especialmente la de los insectos, que es tan abundante y que abarca una amplia distribución geográfica; cuyos representantes tienen diversos ciclos de vida y que además gozan de un gran éxito de colonización en distintos ecosistemas; teniendo presente en que hace falta mucho por descubrir, identificar y conocer. En este sentido la fauna Acridoidea ha sido pobremente estudiada en Panamá, se han realizado algunos estudios taxonómicos, pero son casi nulos los que presentan aspectos biológicos y conductuales de este interesante grupo de insectos.

Los saltamontes son un grupo importante de insectos, con características morfológicas que incluyen especies ápteras, braquípteras o aladas; que habitan en el sotobosque, en el dosel del bosque, a orillas de ríos, también a orillas de áreas costeras, en áreas boscosas o con poca vegetación, cuyos colores van desde oscuros o poco coloridos hasta los que tienen colores llamativos, ya sea en la etapa juvenil o adulta. 
Su alimentación se basa generalmente de tejido vegetal, con frecuencia gramíneas, también pueden incluir algunos cultivos que se producen para la alimentación humana, llegando a causar serios daños a la agricultura, principalmente cuando hay explosiones poblacionales de especies que pueden ser gregarias, las cuales desde tiempos ancestrales han llegado a tener relevancia económica a nivel mundial, ya que resultan ser una plaga importante, para cultivos como: maíz, sorgo, arroz, frijol, entre otros (Zumbado \& Azofeifa, 2018).

Carbonell et al., 2006, citado por Pocco et al., 2010, refiere que especies principalmente de las subfamilias de Acrididae: Melanoplinae y Gomphocerinae y de la familia Romaleidae tienen relevancia económica, ya que ocasionan daños a la agricultura en Argentina.

En Norte y Centroamérica tenemos el caso de Schistocerca spp., esta langosta es la plaga migratoria más destructiva del mundo (FAO, 2020). Schistocerca gregaria, es una plaga endémica de Centroamérica, según datos del Organismo Internacional Regional de Sanidad Agropecuaria (OIRSA) en 1953 causó daños desde México hasta Panamá y desde entonces tiene brotes esporádicos (Usi, 2020); en el 2020 hubo brotes en Centroamérica, pero esta vez la "manga" como comúnmente se le denomina no llegó a Panamá.

En cuanto a los hábitos reproductivos, la estridulación o producción de sonidos con distintas partes del cuerpo es un mecanismo presente en la mayoría de las especies de acridoideos, que tiene como objetivo favorecer el encuentro de los sexos (Aguirre-Segura \& Barranco, 2015). Las hembras depositan sus huevos en el suelo, hojas, troncos, cortezas, hojarasca y ramitas (Zumbado \& Azofeifa, 2018). Tienen la desventaja que sus ootecas son depredadas por un grupo considerable de insectos como, dípteros (sarcofágidos, antómidos, léptidos y bombílidos), coleópteros (cléridos y meloidos), himenópteros (esceliónidos); además, algunos grupos de ácaros (trombídidos y eritreidos) y nemátodos que parasitan a los adultos; también pueden ser infectados por hongos entomopatógenos; todos estos enemigos naturales son importantes a la hora de controlar las mangas que pueden presentarse al darse las condiciones adecuadas para su ocurrencia.

Además, suelen ser presa tanto de vertebrados (mamíferos, aves, reptiles) como de invertebrados (himenópteros esfécidos, arácnidos, 
mántidos, etc), también pueden llegar a ser alimento en diferentes culturas; por lo que son un grupo que se encuentra en la base de las cadenas tróficas de casi todos los ecosistemas donde habitan (AguirreSegura \& Barranco, 2015; Quesada-Béjar et al., 2017; Zumbado \& Azofeifa, 2018).

A continuación, se presenta información de observaciones realizadas tanto in situ como en el cautiverio de aspectos biológicos y conductuales de los saltamontes (Acridoidea).

\section{ACRIDIDAE}

ACRIDINAE

Metaleptea brevicornis (Johannson), 1763, se examinaron hembras que tenían una bolsa de aproximadamente 25 huevos; estos saltamontes se encuentran frecuentemente en áreas abiertas, con gran incidencia de luz y cubiertas por gramíneas (Poacaeae). Se observó que al volar hacen un fuerte sonido "crak-crak-crak", que es típico de la tribu Hylopterygii (Neotrópico), Rowell comunicación personal; a este sonido se le conoce como crepitación y se define como el sonido producido por las alas posteriores durante el vuelo (Lorier et al., 2010).

\section{CYRTACANTHACRIDINAE}

Schistocerca nitens (Thunberg), 1815, de todos los saltamontes de este estudio, esta especie es la especie que realiza una mayor distancia al salir en vuelo de un punto a otro, recordemos que este género puede volar y recorrer grandes extensiones de terreno y que forma mangas perjudiciales para la agricultura; los especímenes fueron colectados en el borde del bosque a orillas de la playa y había hembras con huevos.

\section{GOMPHOCERINAE:}

Amblytropidia trinitatis (Bruner), 1904, encontramos hembras con paquetes de 14 huevos al ser disectadas; estos saltamontes se ven con frecuencia en Poaceae, principalmente Saccharum spontaneum L. (paja canalera, paja blanca, paja de mula ó caña silvestre).

Orphulela punctata (DeGeer), 1773 (Fig.1), es el saltamonte más común de Panamá (Rowell, comunicación personal). Es un saltamonte pequeño, bastante rápido y muchas veces pasa desapercibido, no cae tan fácilmente en la red entomológica y si lo hace, por su tamaño y agilidad de salto se escapa. Al ser disectadas, algunas hembras tenían bolsas con 
30 huevos en su abdomen; esta especie se observa frecuentemente en áreas cubiertas por gramíneas.

\section{LEPTYSMINAE:}

Stenacris fissicauda (Roberts), 1978, se colectaron dentro del sendero pantanoso del Parque Nacional Humedales de San San; fue común encontrarlos en grupos de por lo menos 3 a 4 individuos posados en una misma planta, nunca se observaron solitarios.

Stenopola dorsalis (Roberts \& Carbonell), 1979, colectada en el sendero pantanoso del Parque Nacional Humedales de San San; muchas veces compartían las mismas plantas con Stenacris fissicauda y Stenopola puncticeps.

Stenopola puncticeps (Stål), 1860 (Fig. 2), se encuentra frecuentemente en las orillas de senderos en el sotobosque, pantanos y caminos abiertos, raramente se les vio solitarios; en el dosel del bosque se colectó una pequeña ninfa, posada en una hoja de Apeiba aspera (Tilaceae). Es muy común en los muestreos, encontrándose en casi todos los sitios de colecta.

Rowell (1997a), señala que algunas especies del género Guetaresia, Stenacris y Stenopola se alimentan sólo de algunas monocotiledóneas (Poacaeae y Araceae).

\section{OEDIPODINAE:}

Heliastus sumichrasti Saussure, 1861, se observó durante los meses de octubre a diciembre una gran cantidad de juveniles; esta especie fue encontrada solamente a orillas de los ríos o playas, nunca en senderos ni dentro del bosque; en este sentido, Otte (1984), refiere que esta especie habita terrenos descubiertos, lechos arenosos de ríos y playas; por lo que su alimentación es a base de vegetación relacionada con estos ecosistemas.

\section{OMMATOLAMPINAE:}

Abracris flavolineata (DeGeer), 1773 (Fig. 3), es la segunda especie más abundante en cuanto a distribución (Roberts \& Carbonell, 1981); Rowell (1983), señala que este probablemente sea el chapulín más común y más ampliamente distribuido en los trópicos del Nuevo Mundo, extendiéndose desde el sur de México hasta Argentina. Por lo 
general, en Panamá, es la especie más frecuente en diferentes sitios de colecta (De Gracia \& Cambra, 2002; De Gracia et al., 2011).

Es un saltamonte de mediano tamaño y se le puede capturar con gran facilidad; los machos tienen una longitud hasta de $3.3 \mathrm{~cm}$ y las hembras hasta $4 \mathrm{~cm}$, sus alas son funcionales; las larvas tienen un color críptico, ya sea verde o pardo, superficialmente la larva es muy similar a la de Schistocerca nitens, que a menudo, se encuentra en el mismo hábitat, pero la larva de $A$. flavolineata, puede distinguirse especialmente por su apariencia de "cara aplastada" y por su tamaño un poco menor en todas las etapas; ecológicamente es de interés debido a su formidable éxito para dispersarse a los hábitats interrumpidos y colonizarlos debido a su estrategia de alimentación generalista, aún en bosque de alta diversidad (Rowell, 1983).

Fue común encontrarlos en áreas abiertas cubiertas por gramíneas; además de ser una de las especies cuyas hembras casi siempre al ser disectadas para la preparación del montaje, tenían huevos.

Mycrotylopteryx fusiformis (Rehn), 1905, se colectaron especímenes que se encontraban sobre los $800 \mathrm{msnm}$, en un bosque muy húmedo; con frecuencia estaban en plantas de novio, Impantiens sp. (Balsaminaceae), pero al colocarlos en bolsas ziploc, no comieron de sus hojas.

Rhachicreagra dierythra (Rowell \& Amedegnato), 2000, colectados sobre los $800 \mathrm{msnm}$; se observó mayor cantidad de machos que de hembras. La primera vez que se observó había un grupo de 3 machos en una planta de Hedyosmum bonplandianum (Chloranthaceae), a orilla del camino y con gran incidencia de luz solar; en el laboratorio solamente una pequeña porción de una hoja fue comida, por lo que no podríamos asegurar que esta planta les sirve de alimento, ya que no fue consumida en su totalidad. Puede interpretarse que la comieron, ya sea para probarla o porque tenían hambre o bien no comieron por estrés. La única hembra colectada fue capturada cuando copulaba sobre una hoja seca de una Chloranthaceae. Otros machos se encontraban a orilla del sendero en Hedyosmun bonplandianum, en un área con poca penetración de luz. 


\section{PROCTOLABINAE:}

Esta subfamilia incluye especies tanto del sotobosque como del dosel del bosque.

Adelotettix gigas (Descamps \& Rowell), 1978, esta especie habita en el dosel del bosque, colectada en Apeiba aspera. Es la especie de mayor tamaño dentro de la Proctalabinae.

Ampelophilus olivaceus (Giglio-Tos), 1897, el dato particular para esta especie es que 2 hembras (Fig. 4) estaban parasitadas por el nemátodo Mermis nigrescens (Nematoda: Mermitidae) (Fig. 5), esta observación confirma la literatura que indica que los saltamontes pueden ser parasitados no sólo por otros artrópodos sino también por nemátodos.

Balachowskyacris rhabdotus (Hebard), 1924, puede ser colectado tanto dentro como en el borde del bosque; esta especie fue observada in situ, comiendo una hoja de Piper sp. (Piperaceae).

\section{RHYTIDOCHROTINAE}

Oedalacris lesbiae (Rowell \& Bentos-Pereira), 2005, (Fig. 6), se encontraban a orillas del río Diandummad (Ustupo, Tierra firme) sobre plantas de Pothomorphe peltata Piperaceae (hinojo), comprobando en cautiverio que esta planta les sirve de alimento (Fig. 7), ya que comieron vorazmente las hojas de dicha planta (De Gracia, 2001); estaban junto a otros saltamontes, por ejemplo, Stenopola puncticpes.

\section{ROMALEIDAE}

\section{BACTROPHORINAE}

Esta subfamilia comprende tanto especies del sotobosque como del dosel del bosque. Los saltamontes que habitan el dosel del bosque poseen fémures robustos que favorecen el salto en la copa de los árboles.

Caenolampis robertsi Descamps, 1978 (Fig.8), este saltamontes es habitante del dosel del bosque; las hembras sólo se encuentran en el sotobosque cuando van a ovipositar; por su parte los machos nunca bajan al sotobosque. En el laboratorio, una hembra colocó tres huevos en una sustancia chocolate. 
Lagarolampis gamboensis Rowell, 1999, es otra especie del dosel del bosque, a pesar de que colectamos un macho juvenil en Terminalia amazonia (Combretaceae) y una hembra en Apeiba aspera (Tilaceae), en cautiverio, no se alimentaron de sus hojas.

Mezentia gibbera Stål, 1878, colectada sobre un tronco, estos saltamontes suelen mimetizarse muy bien con estas superficies.

Nautia flavosignata Stål, 1878, es otro bactrophorino habitante del dosel del bosque, colectamos una hembra sobre un helecho arbustivo, fue colocada en una bolsa ziploc para observar en cautiverio su comportamiento; ésta depositó 8 huevos envueltos en una sustancia chocolate (Fig. 9); al cabo de 45-47 días emergieron 6 pequeñas ninfas, confirmando que la hembra baja al sotobosque a ovipositar.

Taeniophora panamae Hebard, 1924, especie del sotobosque, in situ observamos tanto a juveniles como adultos alimentándose de Solanum sp. (Solanaceae).

Zoumolampis bradleyi Rehn, 1929, otra especie habitante del dosel del bosque es un saltamontes braquíptero (Fig.10); se colectaron 2 hembras una en el dosel del bosque y otra en el sotobosque; la que estaba en sotobosque puso 3 huevos cubiertos por una sustancia chocolate, los cuales no llegaron a eclosionar.

\section{ROMALEINAE}

Agriacris tricristata Hebard, 1923, mediante observaciones in situ comprobamos que se alimentan de Solanum sp. (Solanaceae), esta planta les servía tanto de alimento como de refugio; tres hembras fueron colectadas en cópula, en esta planta.

Chromacris psittacus Gerstaecker, 1873, observamos tanto hembras como machos posados en diferentes partes del arbusto Solanum sp., también colectamos especímenes en cópula; in situ comprobamos que se alimentan de dicha planta, llegando a encontrar un arbusto completamente defoliado.

Legua crenulata Stoll, 1813, esta especie la observamos a orillas de una charca, rodeada por gramíneas y pequeñas plantas rastreras. 
Maculiparia rotundata Stål, 1878, es una especie que encontramos frecuentemente en gramíneas.

\section{CONCLUSIONES}

Los resultados de las observaciones tanto en in situ como en cautiverio de los Acridoideos, contribuyen al conocimiento biológico y conductual, y con ello aportan información valiosa a la hora de tomar medidas de control y manejo en este grupo de insectos, debido que de darse las condiciones apropiadas pueden formar mangas que resultan perjudiciales para la agricultura y economía de un país.

La mayoría de los individuos estaban de paso en las plantas donde se encontraron, ya que en el laboratorio pocos comieron sus hojas, probablemente, pudo influir el estrés del cautiverio.

$\mathrm{Al}$ encontrar muchas hembras con masas de huevos, consideramos que se debe a que los muestreos se hicieron en temporada lluviosa, lo que favorece la cópula y oviposición, al encontrarse el suelo suave y húmedo; permitiendo también abundancia de vegetación cuando eclosionen las ninfas.

De acuerdo, a la cantidad de huevos encontrados, podemos decir que las hembras de la Acrididae depositan mayor cantidad, contando un máximo de 30 huevos, con respecto a la Romaleidae (Bactrophorinae) que depositaron un máximo de 8 huevos.

Deben realizarse muestreos más intensivos en el dosel del bosque, para obtener más información, tanto taxonómica como biológica y conductual de los saltamontes de este hábitat casi inexplorado en Panamá.

Hacer observaciones en otras regiones del país pueden llevar a aumentar el conocimiento en cuanto a los aspectos biológicos y conductuales de los saltamontes; ya que hay pocos estudios previos que generalmente se refieren a taxonomía, por lo que en nuestro país, han sido pobremente muestreados y estudiados. 


\section{AGRADECIMIENTOS}

Agradecemos a Publio González, Carlos Vega y Sergio Bermúdez, por su ayuda durante el muestreo; al profesor Luis Carrasquilla de la Universidad de Panamá, por la identificación de las muestras vegetales; al Dr. C.H.F. Rowell, Universidad de Basel, Suiza, por la colaboración en la identificación del material colectado y por sus sabias observaciones; a Vibeke Holdrich, del Instituto Smithsoniano de Investigaciones Tropicales (STRI), proyecto de la grúa del dosel del bosque en Fort Sherman, Colón.

\section{REFERENCIAS}

Aguirre-Segura, A. y P. Barranco. 2015. Clase Insecta Orden Orthoptera. Revista IDE@ - SEA, 46, 1-13.

Bruner, L. 1904-1908. Biología Centrali-Americana, Orthoptera, Vol. II: 65, 243-247; 273-331.

Carbonell, C. S., M.M. Cigliano, y C.E. Lange. 2006. Especies de Acridomorfos (Orthoptera) de Argentina y Uruguay. CD ROM. Publications on Orthopteran Diversity. The Orthopterists' Society at the Museo de La Plata, La Plata.

Cigliano, M.M. y C.E. Lance. 1998. Orthoptera. P. 67-83. En: Morrone, J.J \& S. Coscaron (eds). Biodiversidad de Artrópodos Argentinos. Ediciones Sur Argentina.

DeGeer, C. 1773. Onziéme memoire, des criquets, pp 460-504. In: Memoires pour servir a I'histoire des insects. Stockholm, Tome 3, viii $+697 \mathrm{pp}$.

De Gracia, L. 1. 2001. Acridoidea (Orthoptera: Caelifera) de la vertiente Atlántica del Istmo de Panamá, con observaciones sobre su biología. Tesis de Licenciatura, Universidad de Panamá. 100 págs.

De Gracia L.I. y R.A. Cambra. 2002. Primera contribución al conocimiento de los saltamontes (Orthoptera: Acridoidea) del Parque Nacional Coiba, Panamá. Tecnociencia, 4 (2), 101-109. 
De Gracia, L.I, C.H.F. Rowell y R.A. Cambra. 2011. Diversidad y biología de los saltamontes (Orthptera, Caelífera: Acridomorpha) del Parque Nacional Darién, Estación Rancho Frío, Panamá. Scientia, 21 (2), 47-59.

Descamps, M. 1978. La Faune Dendrophile Néotropicale. II. Revue des Taeniophorini et Ophthalmolampini (Orthoptera Romaleididae). Bulletin du Musséum National d'Histoire Naturelle, N .517 , Zoologie, 355, 371-476.

Descamps, M. y C.H.F. Rowell. 1978. Acridiens des clairiéres de Costa Rica. Diagnoses, signalisations, notes biologiques, polymorphisme (Acridomorpha, Acrididae). Annales de la Société Entomologique de France (N.S.)., 14(3), 351-367.

Food and Agriculture Organization (FAO). 2020. La langosta del desierto. http://www.fao.org/locusts/es/

Gerstaecker, A. 1873. Acridoidea nonnulla nova insigniora. Stettin Entomological Society, 34, 185-197.

Giglio-Tos, E. 1897. Ortotteri raccolti nel Darien dal Dr. E. Festa. III, Acridididae - Gryllidae. Bollettino dei Musei di Zoologia ed Anatomia Comparata della R. Universitá di Torino, 12(301), 1-10.

Hebard, M. 1923. Studies in the Dermaptera and Orthoptera of Colombia, 3, Orthopterous Family Acrididae. Transactions of the American Entomological Society, 49, 165-313.

1924. Studies in the Acrididae of Panama (Orthoptera). Transactions of the American Entomological Society, 50(851), 75-140.

Johannson, B. 1763. Centurio insectorum quam praeside D.D. Car. von Linne propofuit B.J. Calmarienfis. En: Carolia von Line, Amoenitates Academicae seu dissertations variae Physicae, Medicae, Botanicae anthehac seorim editae, vol. 6. $2^{\text {nd }}$ ed. 1789, Erlander.

Lorier E., M.E. Clemente, M.D. García y J.J. Presa. 2010. ECOLOGY, BEHAVIOR AND BIONOMICS El Comportamiento Acústico de 
Fenestra bohlsii Giglio-Tos (Orthoptera: Acrididae: Gomphocerinae). Neotropical Entomology, 39(6), 839-853.

Otte, D. 1984. The North American Grasshoppers Acrididae: Oedipodinae. Harvard University Press, 2, 1-339.

Pocco, M. E., M. P. Damborsky y M. M. Cigliano. 2010. Comunidades de ortópteros (Insecta, Orthoptera) en pastizales del Chaco Oriental Húmedo, Argentina. Animal Biodiversity and Conservation 33 (2), 119129.

Quesada-Béjar, V., M. Nájera, E. Reyes-Novelo y C. GonzálezEsquivel3. 2017. Ortópteros (Caelifera) y sus hongos entomopatógenos en agroecosistemas de maíz en Erongarícuaro, Michoacán* Orthoptera (Caelifera) and its entomopathogens fungi in maize agroecosystems in Erongarícuaro, Michoacán. Revista Mexicana de Ciencias Agrícolas., 8 (6), 1357-1370.

Rehn, J.A.G. 1905. A Contribution to the Knowledge of the Acrididae (Orthoptera) of Costa Rica. Proceeding of the Academy of Natural Sciences, Philadelphia, 67, 400-454.

(1929). Studies in Costa Rican Dermaptera and Orthoptera. New genera and species of Acrididae. Transactions of the American Entomological Society, 44, 321-372.

Roberts, H.R. 1978. A revision of the Tribe Leptysminai except the genus Cylindrotettix (Orthoptera: Acrididae: Leptysminae). Proceeding of the Academy of Natural Sciences, Philadelphia, 129, 33-69.

Roberts, H.R., C.S. Carbonell. 1981. A revision of the neotropical genus Abracris and related genera (Orthoptera, Acrididae, Ommatolampinae). Proceeding of the Academy of Natural Sciences, Philadelphia, 133, 114.

1979. A revision of the genera Stenopola and Cornops (Orthoptera: Acrididae: Leptysminae). Proceeding of the Academy of Natural Sciences, Philadelphia, 131, 104-130. 
Rowell, C.H.F. 1983. Osmilia flavolineata *(Chapulín de raya amarilla, yellow-lined Grasshoppers), pp. 764-765. En: Janzen, D.H. (ed). Costa Rican Natural History. University of Chicago press, 816 pp.

1997a. New and little-known species of Cloephoracris (Orthoptera: Caelifera: Romaleidae) from Central America. Revue Suisse de Zoologie, 106 (1), 3-20.

1997b. New Species of Lagarolampis Descamps (Orthoptera, Caelifera, Acridoidea, Romaleidae, Bactrophorine) from Central America. Revue Suisse de Zoologie, 106 (2), 307-324.

2013. The Grasshoppers (Caelifera) of Costa Rica and Panama. Switzerland: Publications on Orthopteran Diversity.

Rowell, C.H.F. y C. Amédégnato. 2000. New species of Rhachicreagra (Abracrini, Ommatolampinae, Acrididae, Orthoptera). Annales de la Société Entomologique de France, 36(4), 398-401.

Rowell, C.H.F. y A. Bentos-Pereira. 2005. The Rhytidochrotinae of Panama, with new species of Hylopedetes Rehn 1929, Oedalacris Descamps and Amédégnato 1972 and Chiriquacris n.gen. Journal of Orthoptera Research, 14(1), 63-90.

Saussure, H. de. 1861. Orthoptera nova americana (Diagnoses praeliminares). II. Revue et Magasin de Zoologie Pure et Appliquée, 2(13), 156-188.

Stål, C. 1860. Orthoptera species novas descripsit. Pp. 299-350. In:

Konglia Svenska Fregatten Eugenies Resa Omkring Jorden under defäl af C.A. Virigin Ăren 1851-1853, Vetanskapliga Lakttagelser På H.M. Konung Oscar der Forstes Befallining Utgifna af K. Svenska Vetanskaps Akademien, II Zoologie, I Insecta pp. 1-617, Stockholm P.A. Norstedt and Söner, 1858-1868.

1878. Systema Acridiodeorum. Kungliga Svenska VetenskapsAkademiens Handlingar, 5, 1-100.

Stoll, C. 1813. Représentation exactement colorée d'apres nature des spectres ou phasmes, des mantes, des sauterelles, des grillions, des 
criquets et des blattes. Amsterdan, J.C. Sepp et fils, vol. 1,79 pp., 25 pl.; $2,28+42$ pp., $13+23$ pl.

Thunberg, C.P. 1815. Hemipterorum maxillosorum. Genera illustrate. Primisque novis speciebus ditata as descripta. Mémoires de l'Académie Impériale des Sciences de St. Pétersbourg, 5, 211-301.

Usi, E. 2020. Alarma por la llegada de la langosta centroamericana. https://www.dw.com/es/alarma-por-la-llegada-de-la-langostacentroamericana/a-54191970

Zumbado, M. \& D. Azofeifa. 2018. Insectos de Importancia Agrícola. Guía Básica de Entomología. Heredia, Costa Rica. (1ª ed.). Programa Nacional de Agricultura Orgánica (PNAO). 204 pp. 


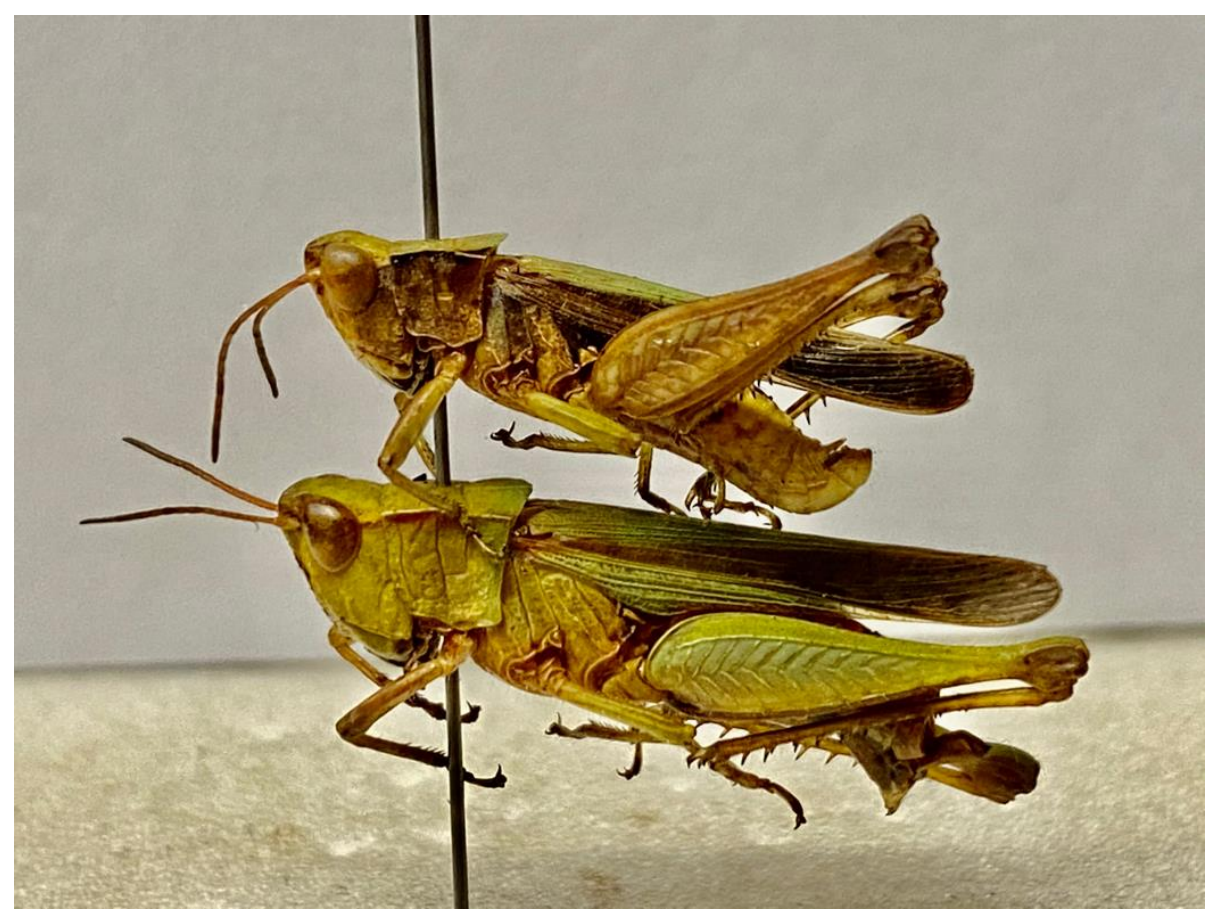

Fig.1. Orphulela punctata, vista lateral.

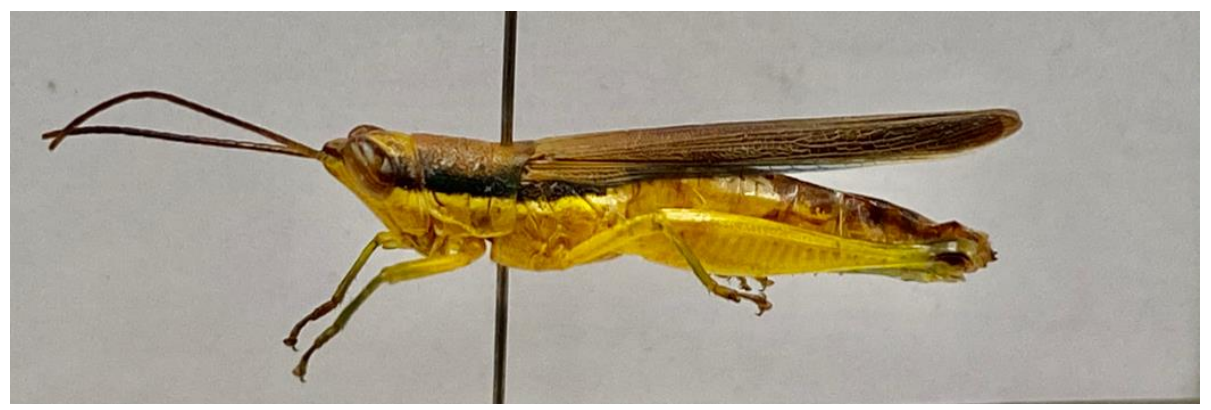

Fig. 2. Stenopola puncticeps, vista lateral. 


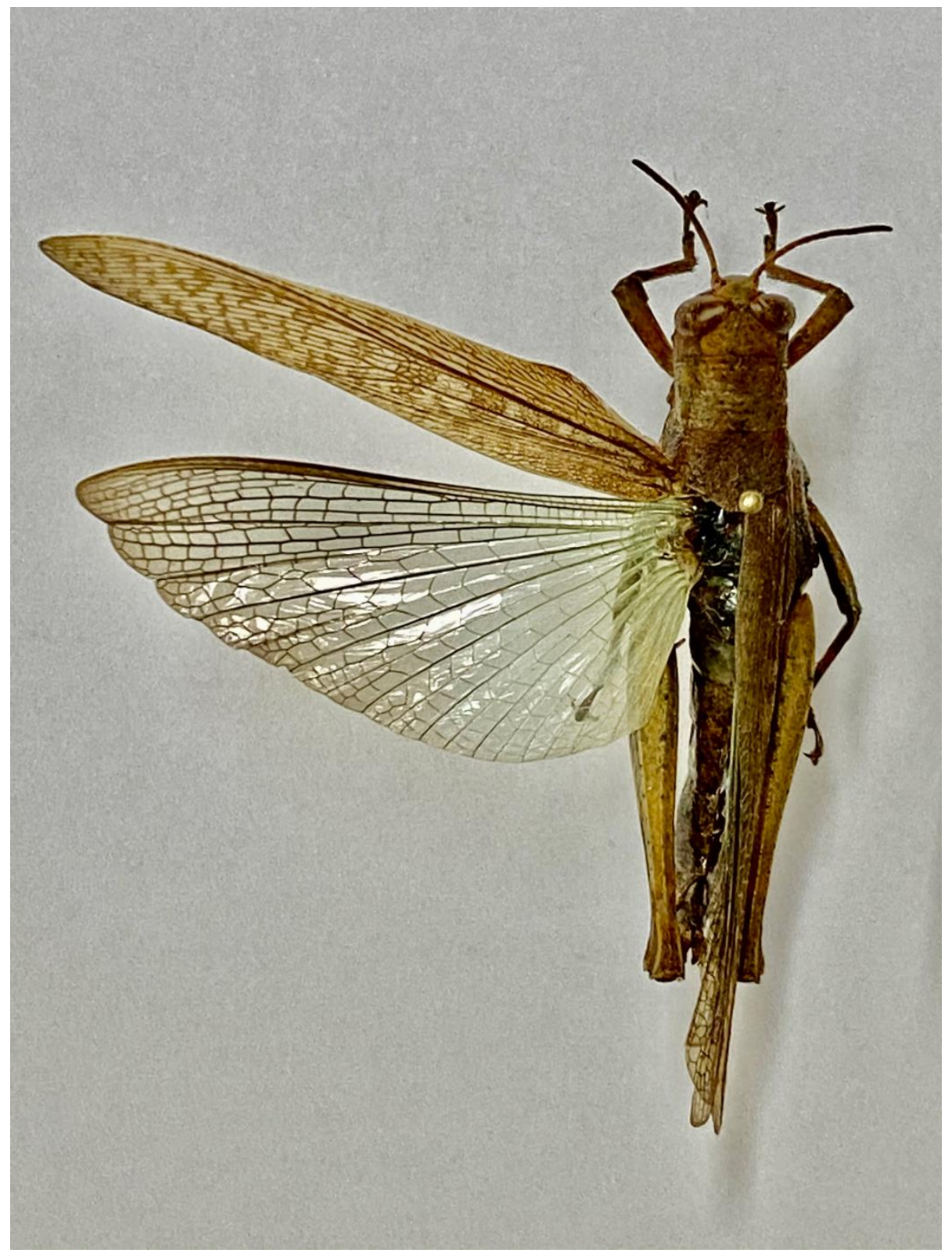

Fig. 3. Abracris flavolineata, vista dorsal. 


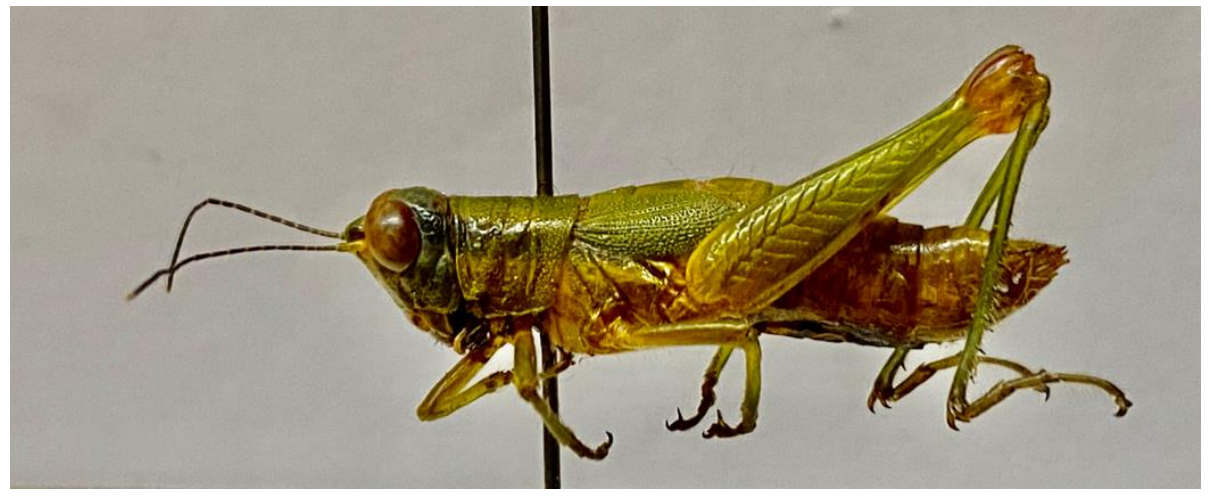

Fig. 4. Ampelophilus olivaceus, vista lateral.

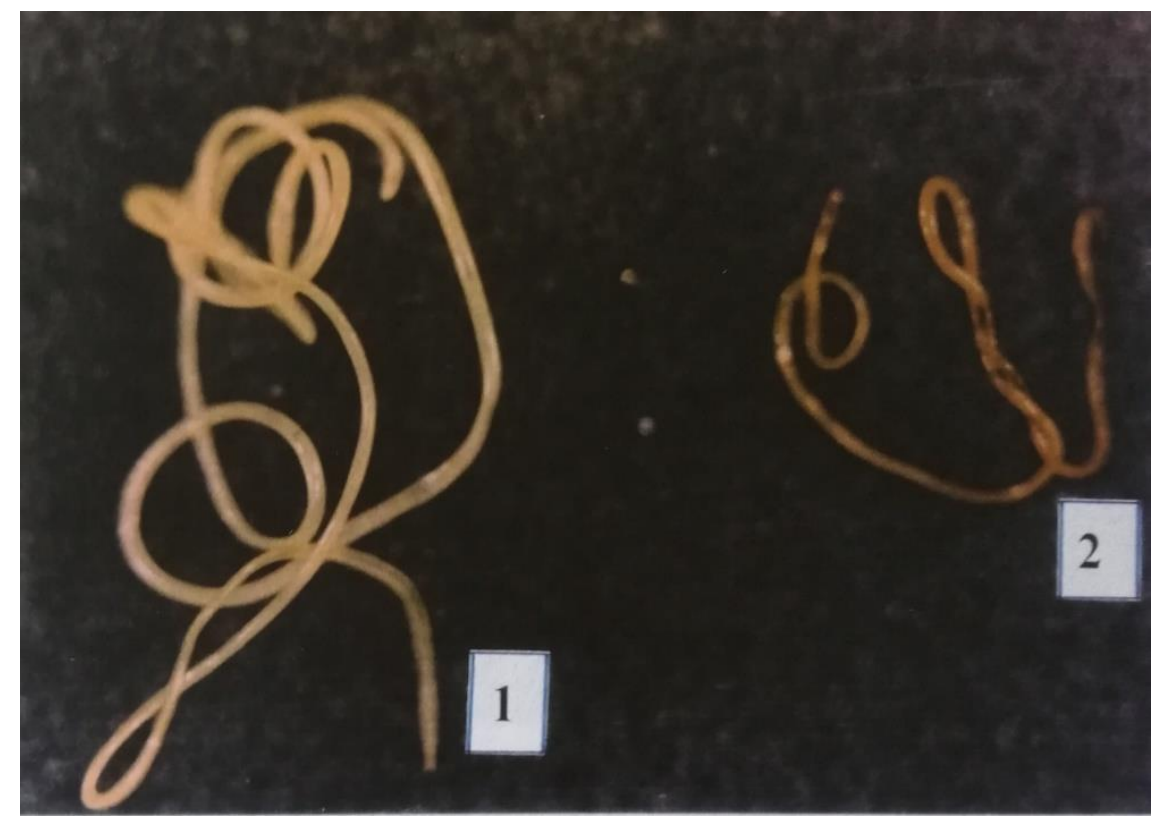

Fig. 5. Mermis nigrescens 


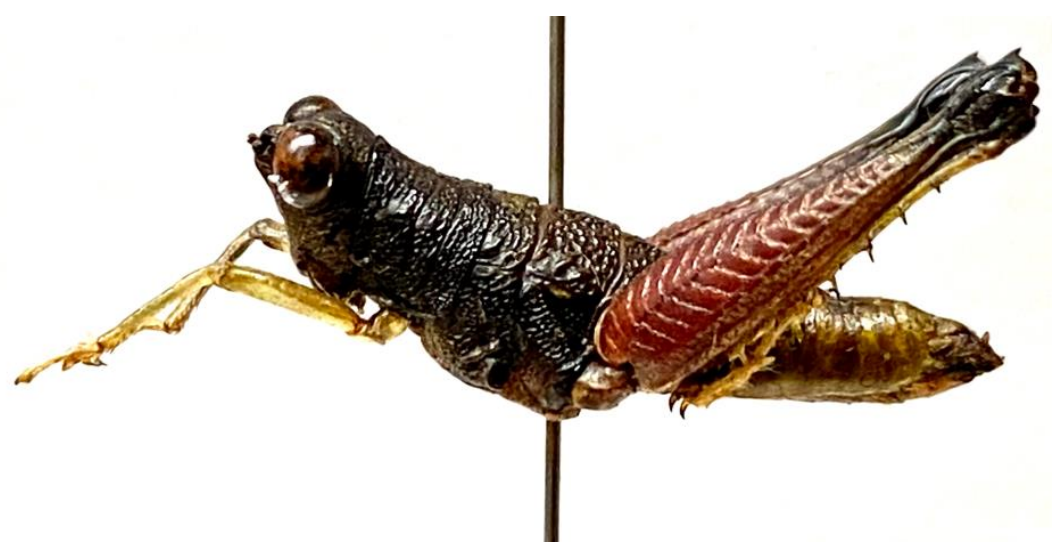

Fig.6. Oedalacris lesbiae, vista lateral.

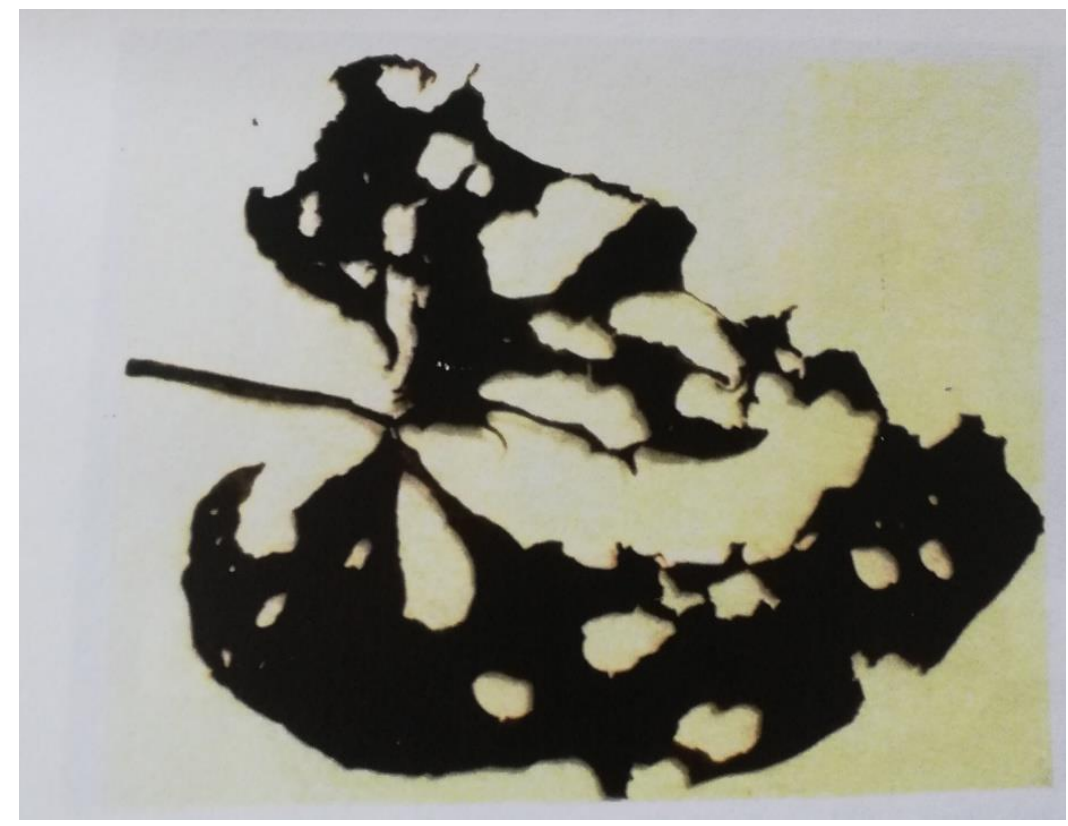

Fig.7. Photomorphe peltata 


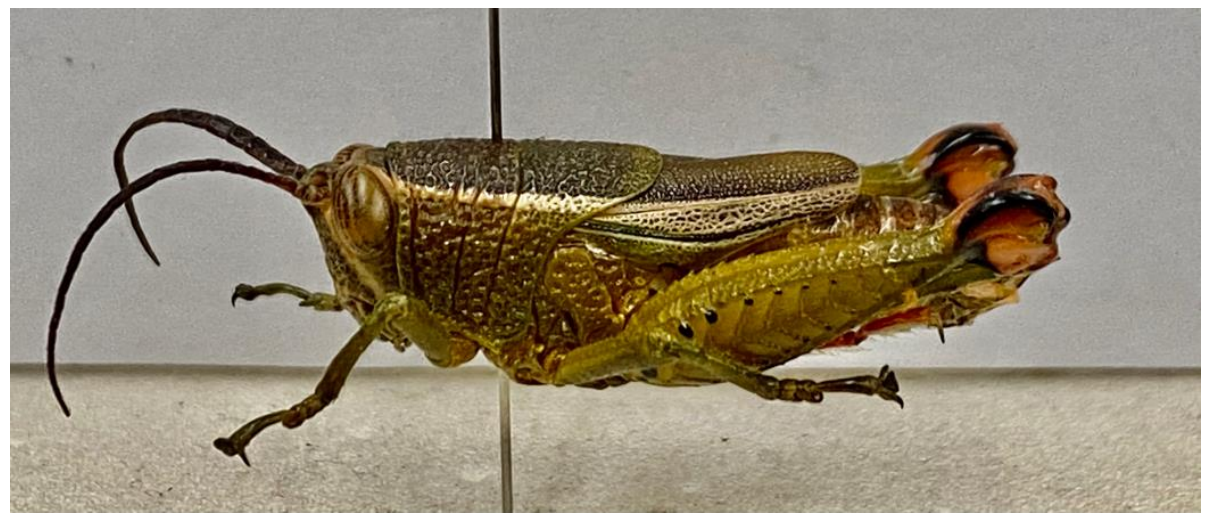

Fig. 8. Caenolampis robertsi, vista lateral.

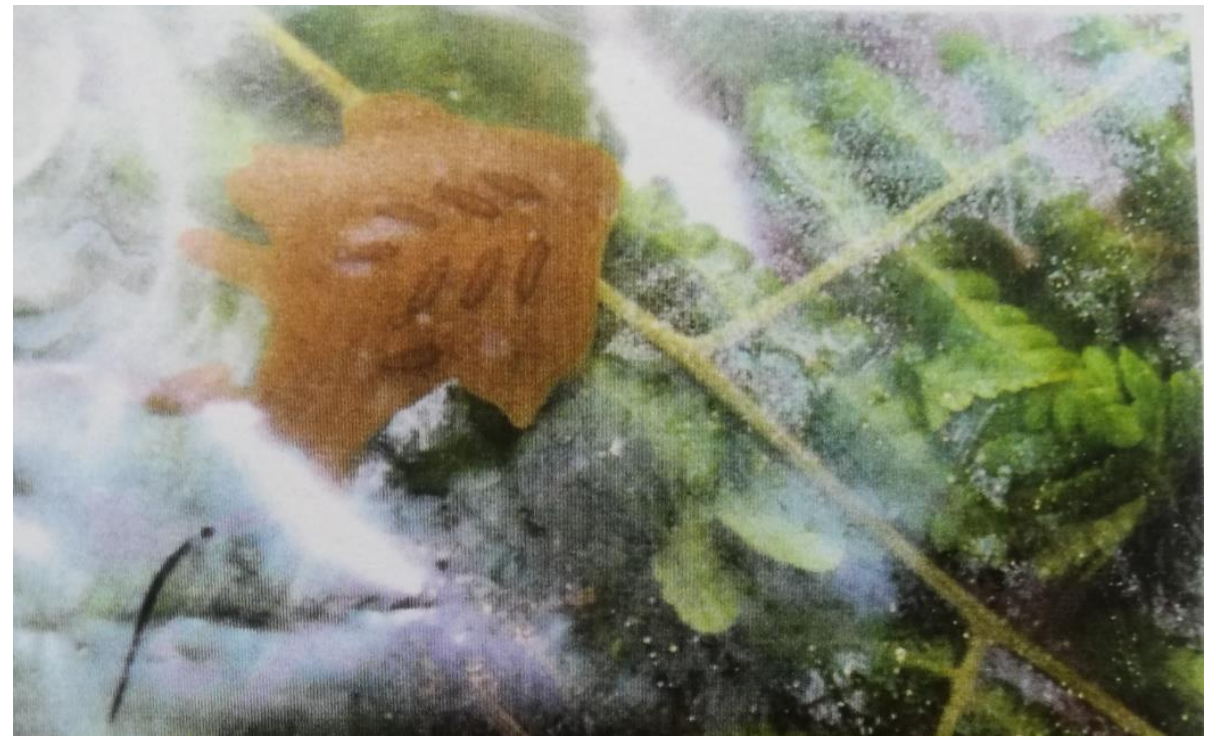

Fig. 9. Huevos de Nautia flavosignata, ovipositados en cautiverio. 


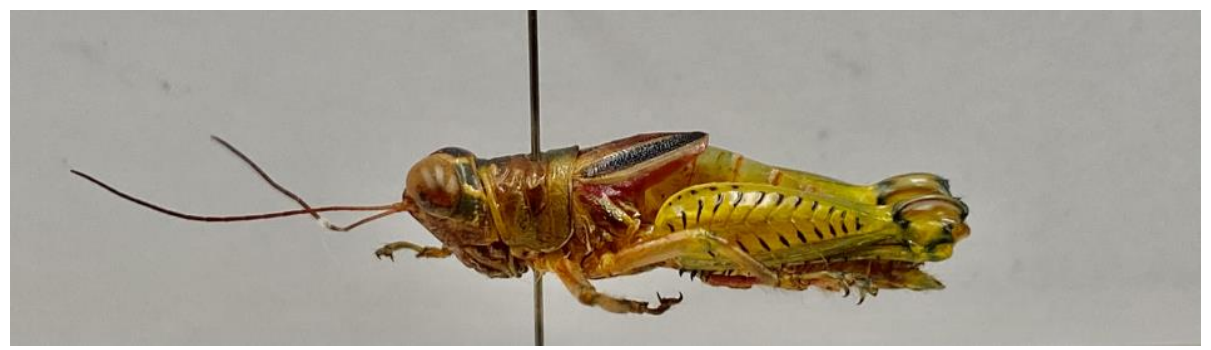

Fig. 10. Zoumolampis bradleyi, vista lateral

Recibido 20 octubre 2020 y aceptado el 07 diciembre 2020 